\title{
Introduction to GPDs and TMDs
}

\author{
Markus Diehl \\ Deutsches Elektronen-Synchroton DESY, Notkestr. 85, 22607 Hamburg, Germany
}

\begin{abstract}
Generalised parton distributions (GPDs) and transverse momentum dependent parton distributions (TMDs) describe complementary aspects of the three-dimensional structure of hadrons. We discuss their relation to each other and recall important theory results concerning their properties and their connection with physical observables.
\end{abstract}

\section{Aspects of nucleon structure}

Among the most intriguing aspects of quantum chromodynamics is the stark contrast between the simplicity of its Lagrangian, formulated in terms of quark and gluon fields, and the complexity of its bound states, hadrons. To understand hadron structure - in particular the structure of the nucleon - in terms of quarks and gluons remains among the most challenging tasks in particle physics. It is also of acute practical importance for the quantitative interpretation of high energy experiments, first and foremost at the Large Hadron Collider.

The parton model describes a fast moving nucleon as a collection of quasi-free quarks, antiquarks and gluons, whose longitudinal momentum distribution is described by parton densities. The formalism of collinear factorisation implements these ideas in QCD and provides the backbone of phenomenology in hadron-hadron and leptonhadron collisions. In several situations it does however not adequately capture all relevant physics, and the conventional parton densities quantify nucleon structure only in a single space dimension.

The distribution of a parton in the plane transverse to the direction of motion of its parent nucleon involves two complementary aspects:

1. the transverse momentum of a parton in the nucleon, quantified by TMDs (transverse momentum dependent distributions), leaves its imprint on the transverse momenta of particles in the final state and can thus be accessed by suitable experimental observables.

2 . the transverse position of a parton in the nucleon can be accessed in suitable exclusive processes where the proton is deflected at small angles. The transverse spatial distribution of the parton can be reconstructed by a Fourier transform from the transverse momentum transferred to the proton, in close analogy with the principle of $X$-ray crystallography. Generalised parton distribution (GPDs) quantify this type of information.

In both cases, the relevant length or momentum scales are typical of non-perturbative dynamics, in contrast to the dimensionless longitudinal momentum fractions on which all types of parton distributions depend. In this sense, TMDs and GPDs carry in a more direct way the imprint of non-perturbative phenomena such as confinement.

Due to the uncertainty principle, transverse momentum and position (along the same direction) cannot be specified simultaneously. However, a unifying description of both aspects can be given using Wigner distributions. These different degrees of freedom and their interplay are discussed in section 2 .

The discussion of "nucleon structure" at the level of quarks and gluons is closely related to the dynamics of the physical processes in which this structure is "probed". To connect nucleon structure with experimental observables in a controlled way, we rely on factorisation formulae proven or conjectured. These formulae express an observable in terms of parton distributions or similar quantities involving non-perturbative dynamics on the one hand and parton-level cross sections or similar quantities involving only dynamics at short distances on the other hand. The latter can be computed in QCD perturbation theory.

In particular the discussion of TMDs shows that the separation of "structure" and "probe" can be surprisingly intricate, to the point of challenging a naive picture of "nucleon structure" itself. Theoretical work aimed at showing exactly when and how information on nucleon structure can be extracted from experimental observables has significantly brought (and continues to push) forward our understanding of QCD in several areas, such as the scale evolution of operator matrix elements, the control of power suppressed (higher twist) contributions, the physical relevance of "unphysical" gluon polarisation, the transition between perturbative and non-perturbative regimes, and the limits of applicability of factorisation. We give a brief overview of such aspects for GPDs in section 3 and for TMDs in section 4 .

As GPDs and TMDs contain information about the longitudinal and at least one of the two transverse directions, they have a much richer spin dependence than the conventional parton densities (the spin dependence 
of which is strongly restricted by rotational invariance). Several GPDs and TMDs describe specific spin-orbit correlations at the parton level and are sensitive to parton orbital angular momentum, which is a crucial ingredient in describing how the overall spin of the nucleon arises from its constituents. In section 5 we make some comments on this topic, which is reviewed in detail in a dedicated contribution to this volume 1 .

For definiteness, we will mostly consider distributions for quarks and antiquarks in the following. Gluon distributions can be discussed in close analogy, with appropriate adaptions.

\section{Space-time and momentum structure}

In this section we review the variables on which different kinds of parton distributions depend. This will allow us to see how the different distributions are related to each other. Any process that probes partons inside a nucleon singles out a particular direction, providing a physical distinction between "longitudinal" and "transverse". This is naturally implemented in the parton model, where one chooses a reference frame in which the hadron under consideration moves fast. One is however not limited to this choice: parton distributions are defined in a covariant way, and one can also discuss them in the hadron rest frame. Of course, the process probing the parton still singles out a particular direction in that frame, so that transverse and longitudinal directions play different roles. Thus, the information one can gain about partons in the proton inevitably breaks manifest three-dimensional rotation invariance. For definiteness, we will in the following consider a reference frame in which the hadron moves fast in the positive $z$ direction (exactly or approximately). A suitable set of coordinates is then given by the light-cone coordinates $v^{ \pm}=\left(v^{0} \pm v^{3}\right) / \sqrt{2}$ and the transverse components $\boldsymbol{v}=\left(v^{1}, v^{2}\right)$ of a given four-vector $v$.

A two-parton correlation function for quarks is defined as the matrix element of a bilinear quark field operator between proton states:

$$
\begin{aligned}
& H(k, P, \Delta)=(2 \pi)^{-4} \int d^{4} z e^{i z k} \\
& \quad \times\left\langle p\left(P+\frac{1}{2} \Delta\right)\left|\bar{q}\left(-\frac{1}{2} z\right) \Gamma q\left(\frac{1}{2} z\right)\right| p\left(P-\frac{1}{2} \Delta\right)\right\rangle .
\end{aligned}
$$

The Dirac matrix $\Gamma$ selects the twist 11 and the parton spin degrees of freedom, and we have omitted labels for the proton spin state. For the moment we put aside field theoretical issues such as the regularisation and renormalisation of the operator and the insertion of a Wilson line between the two quark quark fields. The parton and proton momenta are shown in figure 1, Notice that the on-shell condition for the proton states results in the conditions $P \Delta=0$ and $4 P^{2}+\Delta^{2}=4 m^{2}$, where here and in the following $m$ denotes the proton mass.

\footnotetext{
1 There are several - slightly different - definitions of the term "twist". We will not expand on this topic here and refer to 2 for a detailed discussion.
}

While $H(k, P, \Delta)$ is a smooth function of $\Delta$, the cases where this momentum transfer is zero or not correspond to distinct physical situations:

1. In the forward limit $\Delta=0$ the function appears in the cross section of inclusive processes. Glossing over complications from confinement, one may insert a complete set $|X\rangle\langle X|$ of states between the fields $\bar{q}$ and $q$ in the matrix element (1). This gives essentially the amplitude $\mathcal{A}$ for emitting a quark or antiquark from the proton, with a system of spectator partons $X$ left behind, multiplied by the conjugate $\mathcal{A}^{*}$ of that amplitude as required for the computation of a cross section. The representation as a squared amplitude $\mathcal{A}^{*} \mathcal{A}$ opens the possibility to interpret certain forward distributions as probability densities in the sense of quantum mechanics. Taken literally, this interpretation no longer holds after the regularisation and renormalisation already mentioned, but if taken with due caution it remains a valuable guide for physical intuition.

We note that in the forward limit, it is convenient to take a frame where $\boldsymbol{P}=\mathbf{0}$, so that the proton moves exactly along the $z$ axis.

2. In non-forward kinematics $\Delta \neq 0$ the function appears in the amplitude of exclusive reactions, with an incoming proton of momentum $P-\Delta / 2$ and an outgoing one of momentum $P+\Delta / 2$. The functions in this case are often called "generalised".

In physical observables, the correlation function (1) typically is integrated over one or more components of the four-momentum $k$. Let us review this step by step.

1. After an integral over $k^{-}$, the quark and antiquark fields are evaluated at $z^{+}=0$. This admits a very elegant interpretation in the framework of light-cone quantisation: quark fields are quantised at light-cone time $z^{+}=0$, where they obey the anticommutation relations for free fields and have a Fourier decomposition in terms of creation and annihilation operators for quarks and antiquarks. This may be seen as the field theory implementation of the parton model, where partons are regarded as quasi-free just before they are probed in a physical process. The parton states created or annihilated by the fields have positive plusmomentum, so that depending on the respective signs of $k^{+}-\Delta^{+} / 2$ and $k^{+}+\Delta^{+} / 2$, the matrix element in figure 1 describes the emission and reabsorption of a quark, the emission and reabsorption of an antiquark, or (for $\Delta^{+} \neq 0$ only) the emission or absorption of a quark-antiquark pair (see figure 3 below). At $z^{+}=0$, the representation of the parton correlation function as

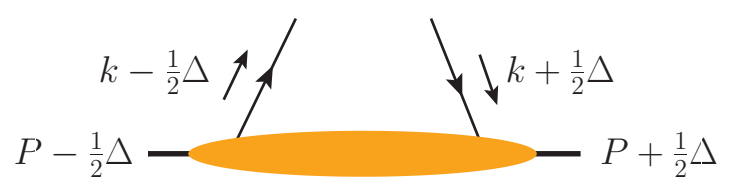

Fig. 1. Momentum assignments in the general quark correlation function (1). 
product $\mathcal{A}^{*} \mathcal{A}$ of an amplitude and its conjugate turns into a product $\psi^{*} \psi$ of a light-cone wave function and its conjugate. More detail is given in the contribution 3. to this volume.

Note that after integration over $k^{-}$the partons no longer have a definite virtuality, in particular they are not on their mass shell. When computing the hard parton-level subprocess for a factorisation formula, one may indeed neglect the parton virtuality (which is much smaller than the hard scale of the process, given e.g. by the photon virtuality in deep inelastic scattering or Drell-Yan production). From the point of view of proton structure, however, the virtuality of partons is essential. In fact, the notion of on-shell partons is at odds with the phenomenon of confinement.

In a field theoretic setting, the integration over $k^{-}$can induce so called rapidity divergences in the matrix element (1), which come from regions where gluons inside a right-moving proton have infinite negative rapidity. Such regions are naturally cut off in a physical process, but in the matrix element they must be excluded by a suitable regulation procedure. The distributions are then dependent on a parameter acting like a rapidity cutoff, and the dependence on this parameter is described by the Collins-Soper equation. We will come back to this in section 4

2. The distributions obtained after an additional integral over $\boldsymbol{k}$ are often called "collinear". This does of course not imply that the partons in the proton have no transverse momentum, but rather that one is not sensitive to their transverse momentum in observables that involve collinear distributions.

The combined integral $\int d k^{-} d^{2} \boldsymbol{k}$ puts the separation between the fields in (11) on the light-cone, $z^{2}=0$, which leads to important simplifications in the field theoretic context. The rapidity divergences mentioned in the previous point disappear after integrating over $\boldsymbol{k}$, so that a rapidity cutoff parameter is no longer needed. In turn, the $\boldsymbol{k}$ integration leads to ultraviolet divergences in the matrix element, which need to be renormalised. They come from regions where partons have infinitely large transverse momentum and virtuality. The dependence of the collinear distribution on the ultraviolet renormalisation scale $\mu$ is described by the familiar DGLAP evolution equations, or by generalised versions of these in the case $\Delta^{+} \neq 0$.

3 . If one also integrates over $k^{+}$, then the parton momentum is fully unspecified and the product of fields in the matrix element (1) becomes a local current. The matrix element is then described by one or more form factors, which depend only on the invariant momentum transfer $\Delta^{2}$ due to Lorentz invariance.

If the current is conserved, the dependence on the renormalisation scale disappears; otherwise it is described by a simple renormalisation group equation with an anomalous dimension depending on the current.

So far our discussion was purely in momentum space. Let us now see how different momentum components in (1) can be traded for position space arguments by a Fourier transform. Again, the transverse and longitudinal directions play very different roles, and for now we focus on the transverse ones. Starting with momentum eigenstates $\left|p\left(P^{+}, \boldsymbol{P}\right)\right\rangle$ of the proton, one can form wave packets

$$
\left|p\left(P^{+}, \boldsymbol{b}\right)\right\rangle \propto \int d^{2} \boldsymbol{P} e^{-i \boldsymbol{P} \boldsymbol{b}}\left|p\left(P^{+}, \boldsymbol{P}\right)\right\rangle,
$$

which are localised at position $\boldsymbol{b}$ in the transverse plane. They are indeed eigenstates of a transverse position operator 4, which means that a relativistic particle of mass $m$ can be localised exactly in two dimensions. (In all three dimensions, this is only possible up to ambiguities of the order of its Compton wave length $1 / \mathrm{m}$ ).

To understand what this localisation means for a spatially extended object like the proton, we consider socalled transverse boosts, which form a subgroup of the Lorentz group and transform a vector $k$ as

$$
k^{+} \rightarrow k^{+}, \quad \boldsymbol{k} \rightarrow \boldsymbol{k}-k^{+} \boldsymbol{v},
$$

where $\boldsymbol{v}$ is a fixed vector characterising the transformation. Notice the analogy between these transverse boosts and the familiar Galilean transformations in nonrelativistic mechanics, which are obtained from (3) by replacing $k^{+}$with the mass $m$ of the particle. The relativistic analogue of the centre-of-mass for a composite system is thus the centre of plus-momentum, $\boldsymbol{b}=\sum_{i} k_{i}^{+} \boldsymbol{b}_{i} / \sum_{i} k_{i}^{+}$. This is the meaning of $\boldsymbol{b}$ in (2).

As already mentioned, a quark field operator $q\left(z^{-}, \boldsymbol{z}\right)$ at $z^{+}=0$ can be decomposed on annihilation and creation operators for parton states. From matrix elements of bilinear field operators at $z^{+}=0$ between the proton states in (2) we can thus define so-called impact parameter distributions, which describe the transverse spatial distribution of partons inside a proton that is localised in the transverse plane [5]. These distributions are obtained from their momentum space counterparts by a two-dimensional Fourier transformation w.r.t. the momentum transfer $\boldsymbol{\Delta}$.

Let us take a closer look at the relation between transverse momentum and position variables. The Fourier transform

$$
q\left(z^{-}, \boldsymbol{k}\right)=\left.\int d^{2} \boldsymbol{z} e^{-i \boldsymbol{k} \boldsymbol{z}} q\left(z^{-}, \boldsymbol{z}\right)\right|_{z^{+}=0},
$$

contains the annihilation operator for a quark with transverse momentum $\boldsymbol{k}$, and for the bilinear operator needed to form a quark density we have

$$
\begin{aligned}
& \bar{q}\left(z_{2}^{-}, \boldsymbol{k}_{2}\right) \Gamma q\left(z_{1}^{-}, \boldsymbol{k}_{1}\right) \\
& \quad=\int d^{2} \boldsymbol{z}_{2} d^{2} \boldsymbol{z}_{1} e^{i\left(\boldsymbol{k}_{2} \boldsymbol{z}_{2}-\boldsymbol{k}_{1} \boldsymbol{z}_{1}\right)} \bar{q}\left(z_{2}^{-}, \boldsymbol{z}_{2}\right) \Gamma q\left(z_{1}^{-}, \boldsymbol{z}_{1}\right) .
\end{aligned}
$$

Rewriting the Fourier exponent as

$$
\begin{aligned}
\boldsymbol{k}_{2} \boldsymbol{z}_{2}-\boldsymbol{k}_{1} \boldsymbol{z}_{1}= & \frac{1}{2}\left(\boldsymbol{z}_{2}+\boldsymbol{z}_{1}\right)\left(\boldsymbol{k}_{2}-\boldsymbol{k}_{1}\right) \\
& +\frac{1}{2}\left(\boldsymbol{k}_{2}+\boldsymbol{k}_{1}\right)\left(\boldsymbol{z}_{2}-\boldsymbol{z}_{1}\right),
\end{aligned}
$$

we can read off the relation between Fourier conjugate variables:

average position $\leftrightarrow$ momentum difference, average momentum $\leftrightarrow$ position difference, 


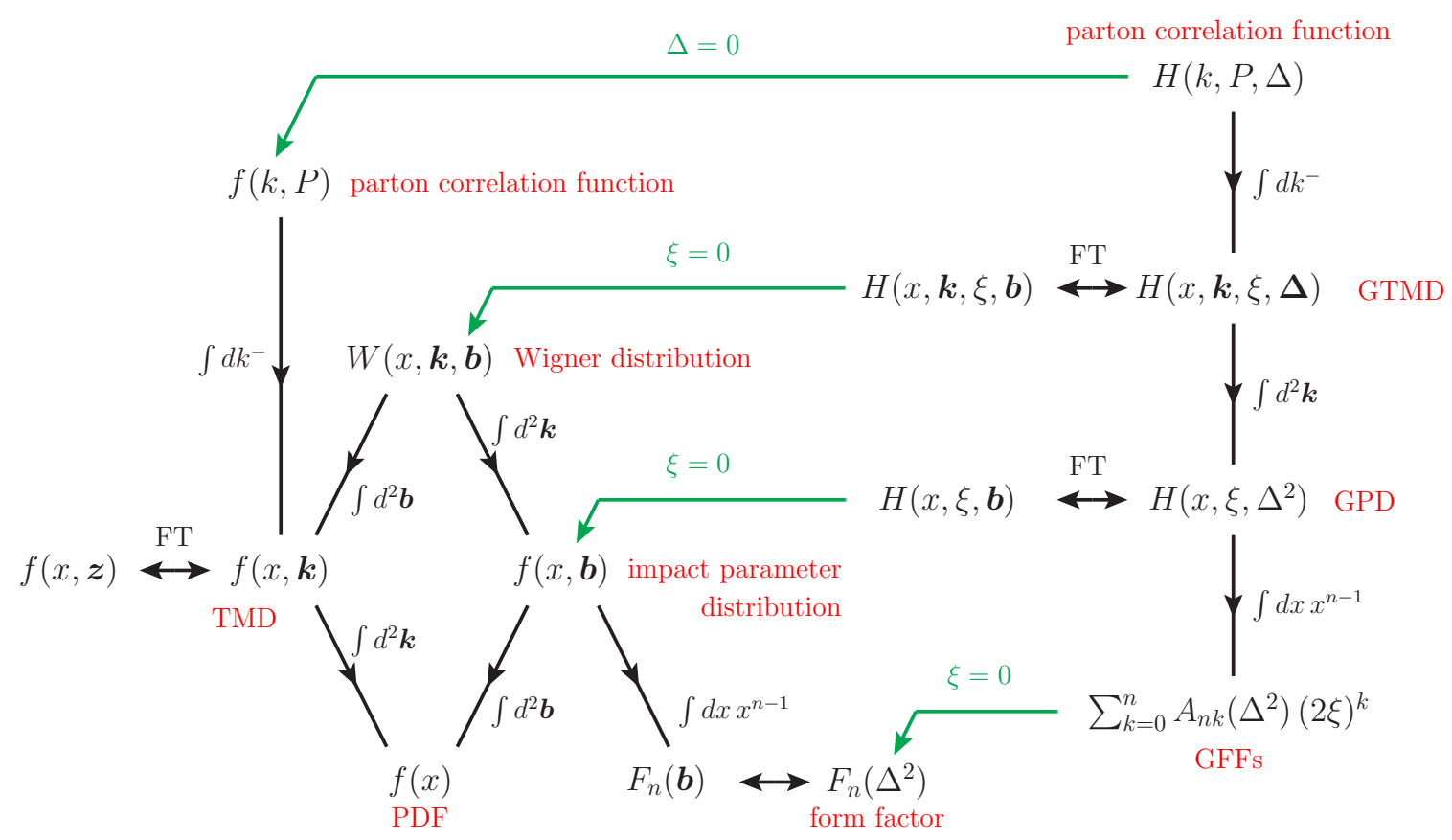

Fig. 2. Selected quantities that can be derived from the fully differential two-quark correlation function $H(k, P, \Delta)$ defined in (11). Double arrows marked by "FT" denote a Fourier transform between $\boldsymbol{\Delta}$ and $\boldsymbol{b}$ or between $\boldsymbol{k}$ and $\boldsymbol{z}$. Fractions of plusmomentum (commonly called "longitudinal momentum fractions") are written as $x=k^{+} / P^{+}$and $2 \xi=-\Delta^{+} / P^{+}$. The invariant momentum transfer can be expressed in terms of longitudinal and transverse variables as $\Delta^{2}=-\left(4 \xi^{2} m^{2}+\Delta^{2}\right) /\left(1-\xi^{2}\right)$. Only kinematic arguments of the functions are given, while the scales introduced by ultraviolet renormalisation $(\mu)$ of by the regulation of rapidity divergences $(\zeta)$ are suppressed. As discussed in the text, the integrals $\int d k^{-}$and $\int d^{2} \boldsymbol{k}$ cannot be taken literally but must be supplemented with a regularisation procedure.

where "average" and "difference" refer to the right and left hand sides of figure 1 or equivalently to the light-cone wave function $\psi$ and its conjugate $\psi^{*}$.

After these general considerations, we can take a closer look at the different distributions that can be obtained from the general two-quark correlation function in (1). A selection of them is shown in figure 2, Let us start at the top of the hierarchy.

1. In the forward limit $\Delta=0$, parton correlation functions that are not integrated over any component of $k$ (called "doubly" or "fully unintegrated" distributions) have been discussed in the context of evolution at small $x$ [6] and with the aim of having an exact description of final-state kinematics $[7,8$. Under the name of "beam functions", they have also been introduced in soft-collinear effective theory (SCET) for the resummation of large logarithms in observables sensitive to the proton remnants (called "beam jets") 9, 10, 11. In that case, distributions differential in $k^{-}$but integrated over $\boldsymbol{k}$ are referred to as beam functions as well. The considerations in [6] and $9,10,11$ focus on the region of large parton virtuality $k^{2}$ and compute the unintegrated distributions in terms of conventional parton distribution functions (PDFs), an aspect we will discuss in more detail for TMDs in section 4 .

A detailed analysis of factorisation with unintegrated distributions has been given for semi-inclusive deep inelastic scattering (SIDIS) in [8]. For hadron-hadron collisions there are strong arguments that this type of factorisation generically fails, due to soft gluon exchange between the spectator partons in each hadron [12,13. In kinematics referred to as the Glauber region, these soft interactions "tie together" the two hadrons in a way that prevents one from describing the non-perturbative dynamics by matrix elements that pertain to only one hadron and not to both. To establish factorisation, one has to show that (after appropriate approximations) gluon exchange in the Glauber region cancels in the observable at hand.

Not being integrated over any momentum component, parton correlation functions retain manifest Lorentz invariance (provided that one is careful not to forget auxiliary vectors required for their field theoretical definition). They can therefore be used to classify and relate different distributions that descend from them. Examples are given in [14] for $\Delta=0$ and in [15] for $\Delta \neq 0$.

2. Wigner distributions depend on the average momentum and the average position of the quark. From the uncertainty principle it is clear that they cannot represent joint probabilities in these two variables, but integrating over any one of them, one obtains a probability in the other.

The most straightforward interpretation of these distributions is in the forward limit $\xi=0$ of longitudinal momentum. Integrating the Wigner distribution 
$W(x, \boldsymbol{k}, \boldsymbol{b})$ in figure 2 over $\boldsymbol{b}$ one obtains the TMD $f(x, \boldsymbol{k})$, which specifies the probability density of finding a parton with longitudinal momentum fraction $x$ and transverse momentum $\boldsymbol{k}$, whilst integration over $\boldsymbol{k}$ yields the impact parameter distribution $f(x, \boldsymbol{b})$, which gives the probability density of finding a parton with longitudinal momentum fraction $x$ at a transverse distance $\boldsymbol{b}$ from the centre of momentum of the proton. There is no process known where the Wigner distributions just described would be directly accessible to experiment. It is amusing to note that more complicated Wigner distributions do appear in the cross section for double parton scattering - a process where in a single proton-proton collision two pairs of partons initiate two separate hard scatters [16]. These double parton distributions $F\left(x_{1}, x_{2}, \boldsymbol{k}_{1}, \boldsymbol{k}_{2}, \boldsymbol{b}_{1}, \boldsymbol{b}_{2}\right)$ are defined in analogy to $W(x, \boldsymbol{k}, \boldsymbol{b})$, but with two bilinear quark operators instead of one. The cross section involves the product

$$
F\left(x_{1}, x_{2}, \boldsymbol{k}_{1}, \boldsymbol{k}_{2}, \boldsymbol{b}_{1}, \boldsymbol{b}_{2}\right) F\left(\bar{x}_{1}, \bar{x}_{2}, \overline{\boldsymbol{k}}_{1}, \overline{\boldsymbol{k}}_{2}, \overline{\boldsymbol{b}}_{1}, \overline{\boldsymbol{b}}_{2}\right)
$$

of two such distributions, one for each proton. It is integrated over the transverse variables with constraints $\boldsymbol{k}_{1}+\overline{\boldsymbol{k}}_{1}=\boldsymbol{q}_{1}$ and $\boldsymbol{k}_{2}+\overline{\boldsymbol{k}}_{2}=\boldsymbol{q}_{2}$, where $q_{1}$ and $q_{2}$ are the momenta of the particles produced in one and the other hard scatter. The additional constraint $\boldsymbol{b}_{1}-\boldsymbol{b}_{2}=$ $\overline{\boldsymbol{b}}_{1}-\overline{\boldsymbol{b}}_{2}$ ensures that the two parton pairs in each proton can initiate two separate short-distance processes.

Because Wigner distributions do not represent probabilities, they need not be positive, which makes an intuitive interpretation somewhat difficult. Using an appropriate smearing procedure, one can obtain socalled Husimi distributions, which are positive definite and can be interpreted as probabilities without contradicting the uncertainty principle. They have been discussed in the context of nucleon structure in [17.

3. The preceding discussion referred to Wigner distributions that depend on momenta and positions in the transverse plane whilst in the longitudinal direction the momentum representation is kept. This "mixed" representation is useful in many contexts (for instance in small- $x$ physics), and it allows one to keep a close connection to the parton model picture, where hadrons and partons move fast in the longitudinal direction. However, one may also Fourier transform longitudinal momentum variables. Three-dimensional Wigner functions have been defined in 18 by a Fourier transform of GTMDs (see figure 2) w.r.t. the momentum transfer $\left(\Delta^{1}, \Delta^{2}, \Delta^{3}\right)$ in the brick-wall frame, where $\Delta^{0}=$ 0 . The interpretation of these quantities is similar to the familiar interpretation of Fourier transformed form factors as three-dimensional spatial densities 19, and it is subject to the same limitations due to special relativity (see our comment above). A different threedimensional representation has been proposed in [20, considering the scattering amplitude $\mathcal{A}(\boldsymbol{\Delta}, \xi)$ for deeply virtual Compton scattering (which is closely related to GPDs) and performing a Fourier transform w.r.t. $\Delta$ and $\xi$. The resulting spatial distribution in the longi- tudinal direction is reminiscent of an optical diffraction pattern.

The Fourier transform w.r.t. the average momentum fraction $x$ has been studied in particular for distributions integrated over $\boldsymbol{k}$, that is for GPDs and PDFs. It gives matrix elements of operators with all fields separated along the light-cone, whose ultraviolet renormalisation can be discussed independently of the hadron momenta. This provides deep insight into the apparently different scale evolution of PDFs and of meson distribution amplitudes, which are matrix elements of the same operators between a meson state and the vacuum. The evolution equations relevant for GPDs turn out to interpolate between these two cases [21,22].

4. TMDs can be measured in a variety of reactions in lepton-proton and proton-proton collisions, where a final-state particle is observed with a transverse momentum much smaller than the hardest scale in the process (such as the photon virtuality in SIDIS or Drell-Yan production). The measured transverse momentum typically results from the convolution of two transverse-momentum dependent quantities, such as a parton distribution and a fragmentation function in SIDIS or two parton distributions in the Drell-Yan process.

This transverse-momentum convolution becomes an ordinary product after a Fourier transform; the resulting distribution $f(x, \boldsymbol{z})$ in figure 2 is often called $f(x, \boldsymbol{b})$ in the literature (a notation reserved for impact parameter distributions here). This representation has also the advantage that Collins-Soper evolution in rapidity (see above) is at fixed $\boldsymbol{z}$, whereas in transversemomentum space it involves again a convolution. Notice that $f(x, \boldsymbol{z})$ does not have a simple probability interpretation, $\boldsymbol{z}$ being the difference between the parton position in the light-cone wave function and its conjugate according to our analysis above.

5. GPDs $H\left(x, \xi, \Delta^{2}\right)$ are accessible in suitable exclusive processes, in which the invariant momentum transfer $\Delta^{2}$ to the proton is much smaller than the hard scale $Q^{2}$, which is often given by the virtuality of a photon. Example processes are deeply virtual Compton scattering (DVCS), $\gamma^{*} p \rightarrow \gamma p$, and exclusive meson production $\gamma^{*} p \rightarrow M p$, both measurable in lepton-proton collisions. A close analogue of DVCS is timelike Compton scattering (TCS), $\gamma p \rightarrow \gamma^{*} p$, where the timelike photon decays into a lepton-antilepton pair. Whereas $\Delta$ is directly measurable, the average parton momentum fraction $x$ always appears convoluted with a function representing the hard-scattering process. The corresponding deconvolution problem is perhaps the most difficult aspect in extracting information about GPDs from experimental data.

The analogous functions that are also differential in the transverse momenta of the partons are called generalised TMDs (GTMDs). Whilst the appearance of GPDs in exclusive scattering amplitudes can be established with great theoretical rigour 23,24, GTMDs have only been used in a phenomenological spirit, in or- 

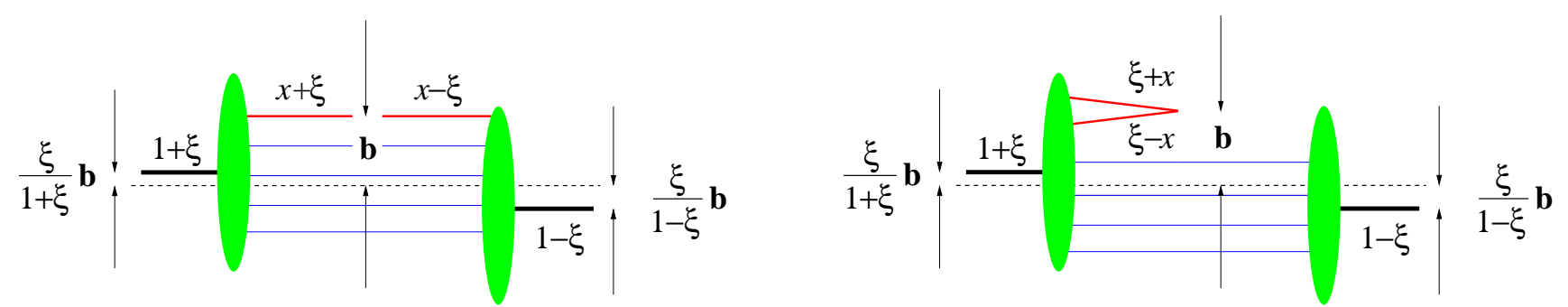

Fig. 3. Impact parameter space representation of a GPD at nonzero skewness variable $\xi$ in the regions $\xi<x<1$ (left) and $|x|<\xi$ (right). $\xi$ is taken to be positive, as appropriate for the processes in which GPDs are known to occur. The overall centre of plus-momentum shifts because of the transfer of plus momentum to the proton. The case $-1<x<-\xi$ (not shown) is analogous to $\xi<x<1$, with an antiquark carrying momentum fraction $-x+\xi$ in the wave function and momentum fraction $-x-\xi$ in the conjugate wave function.

der to quantify subleading corrections in $1 / Q$ to DVCS and exclusive meson production 25. It is not clear to which extent this could be made more rigorous, and even in the setting just mentioned, the average transverse parton momentum $\boldsymbol{k}$ appears under an integral and cannot be reconstructed in a direct manner.

6. A Fourier transform of GPDs (or GTMDs) w.r.t. the transverse part $\boldsymbol{\Delta}$ of the momentum transfer yields distributions depending on the impact parameter $\boldsymbol{b}$, which quantifies the distance of a transversely localised parton from the centre of momentum of the proton. The exclusive processes in which GPDs (or GTMDs) appear involve a finite longitudinal momentum transfer to the proton, so that the corresponding distributions are probed at nonzero $\xi$. This complicates somewhat the interpretation of $H(x, \xi, \boldsymbol{b})$, which has interference character as far as the longitudinal momentum fractions are concerned, as shown in figure 3. Only when one takes the limit $\xi=0$ of this distribution does one obtain the impact parameter distribution $f(x, \boldsymbol{b})$ with a density interpretation. Likewise, one obtains the Wigner distribution $W(x, \boldsymbol{k}, \boldsymbol{b})$ as the $\xi=0$ limit of a Fourier transformed GTMD.

7. Integrating TMDs over $\boldsymbol{k}$ or impact parameter distributions over $\boldsymbol{b}$, one obtains the usual collinear PDFs. Among all quantities discussed so far, the range of processes where these distributions can be studied experimentally is largest. Correspondingly, our knowledge about them is very advanced and often in the realm of precision physics. However, even for PDFs some aspects remain poorly known, such as the distributions of strange quarks and antiquarks or the distribution of longitudinally polarised gluons.

8. Taking Mellin moments $\int d x x^{n-1}$ of a GPD, one obtains a sum over the form factors of a local current, which are often called generalised form factors (GFFs) in this context. As a consequence of Lorentz invariance, they are weighted by powers of $\xi$ in the sum, which strongly constrains the interplay between the $x$ and $\xi$ dependence of the original GPD. This polynomiality property plays a prominent role in the construction of consistent GPD parametrisations.
Hadron matrix elements of local currents in the spacelike region can readily be continued to Euclidean spacetime and thus be computed in lattice QCD. This opens the way to obtain genuinely non-perturbative information about GPDs from first principles and is complementary to model building efforts. A detailed review of this field is given in [26].

Evaluating the Mellin moments of GPDs in the $\xi=0$ limit and performing a two-dimensional Fourier transform to impact parameter space, one obtains the Mellin moments of the impact parameter distribution $f(x, \boldsymbol{b})$, as seen in figure 2 One can thus interpret elastic form factors as two-dimensional densities in the impact parameter plane, with the information about the longitudinal parton momentum being condensed into an average with weight $x^{n-1}$. This provides a parton-based alternative to the representation of form factors as Fourier transformed three-dimensional densities (see above) and has led to surprising insights when applied to the electromagnetic proton and neutron form factors, which are rather well known experimentally [27].

\section{More on GPDs}

In this section we take a closer look at the theory that connects GPDs with exclusive scattering processes. The factorisation of dynamics into perturbative and non-perturbative quantities happens at the level of the scattering amplitude. Schematically, a GPD factorisation formula reads

$$
\begin{aligned}
\mathcal{A} & \left(\xi, \Delta^{2}, Q^{2}\right) \\
& =\sum_{i} \int_{-1}^{1} d x C_{i}(x, \xi ; \log (Q / \mu)) H_{i}\left(x, \xi, \Delta^{2} ; \mu\right)
\end{aligned}
$$

where $C_{i}$ represents the hard-scattering process, $H_{i}$ is a GPD and the sum is over the relevant parton types $i$. Example graphs are shown in figure 4. The support region of the integral over $x$ includes the different regimes discussed in the previous section (see figure 3). In the present section, we gloss over the fact that there are GPDs with different dependence on the spin of the partons and the protons. 

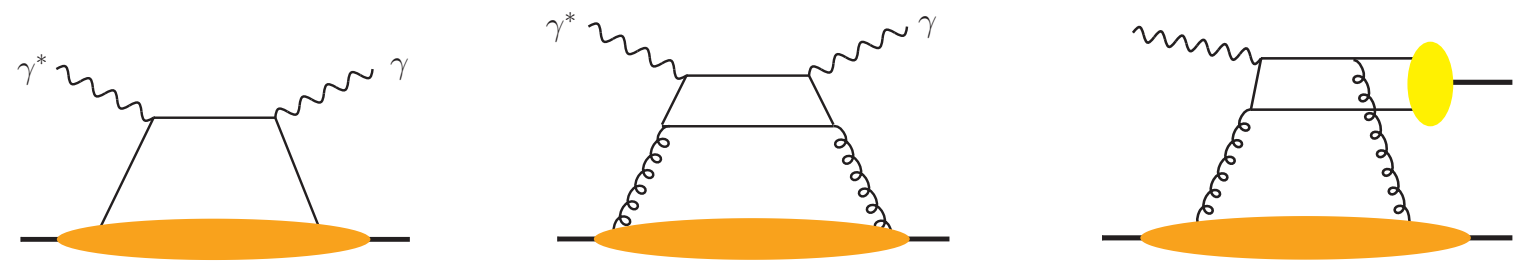

Fig. 4. Example graphs for DVCS (left and centre) and for exclusive vector meson production (right). The lower blob denotes a GPD, and the upper blob in the right panel denotes the meson distribution amplitude.

At lowest order in $\alpha_{s}$, the hard-scattering kernels for DVCS, TCS and meson production are linear combinations of $1 /(\xi-x-i \epsilon)$ and $1 /(\xi+x-i \epsilon)$. This gives a nontrivial convolution for $\operatorname{Re} \mathcal{A}$ while $\operatorname{Im} \mathcal{A}$ involves $H\left(x, \xi, \Delta^{2}\right)$ at the special points where $x= \pm \xi$. At higher orders, logarithms of $Q / \mu$ appear in $C_{i}$, where $Q$ is the physical hard scale. The dependence on the factorisation scale $\mu$ cancels between $C_{i}$ and $H_{i}$ to the order in $\alpha_{s}$ considered, just as in the case of inclusive processes with PDFs. As already mentioned, the separate dependence of GPDs on $x$ and $\xi$ cannot be directly inferred from the amplitude: it is only through the interplay between $x$ and $\mu$ in the scale evolution of $H_{i}$ (combined with the dependence of $C_{i}$ on $Q / \mu$ ) that this aspect of GPDs can be constrained from measurements. The evolution equation for GPDs has the form

$$
\begin{aligned}
& \frac{d}{d \log \mu} H_{i}\left(x, \xi, \Delta^{2} ; \mu\right) \\
& \quad=\sum_{j} \int_{-1}^{1} d \hat{x} V_{i j}\left(x, \hat{x}, \xi ; \alpha_{s}(\mu)\right) H_{j}\left(\hat{x}, \xi, \Delta^{2} ; \mu\right) .
\end{aligned}
$$

Notice that evolution changes the $x$ dependence but is local in the variables $\xi$ and $\Delta^{2}$, which are fixed by the hadron momenta in the matrix element defining the GPD. In the region $|x|>|\xi|$, evolution acts in a similar way as the familiar DGLAP evolution of PDFs, whereas for $|x|<|\xi|$ it is similar to the ERBL evolution of meson distribution amplitudes. Correspondingly, one often calls the respective $x$ ranges the DGLAP or ERBL regions.

The evolution kernels $V_{i j}$ are known at two-loop order, matching the available two-loop accuracy of the hardscattering coefficients $C_{i}$ for light quarks and gluons in DVCS. One loop corrections to $C_{i}$ are available for meson production and for TCS, as well as for DVCS with a heavy-quark loop. Detailed information and references can be found in the review [22], apart for the more recent result [28].

The hard-scattering mechanism selects certain polarisation combinations for the photons and/or mesons in the process, so that a twist-two factorisation formula like (8) only holds for selected helicity amplitudes. Other helicity amplitudes are suppressed by one or two orders in $\Lambda / Q$, where $\Lambda$ represents the scale of non-perturbative dynamics, as well as the proton mass $m$ and the kinematical scale $|\boldsymbol{\Delta}|$. In the case of DVCS and TCS, the leading amplitudes are for transverse $\gamma^{*}$ polarisation, whereas those for longitudinally polarised $\gamma^{*}$ are suppressed by $|\boldsymbol{\Delta}| / Q$. They can be factorised into hard-scattering coefficients and generalised parton distributions of twist three. We shall not review these in more detail here, but mention that certain twist-three distributions are obtained not from the twoparton correlation function (10) but from its analogue with an additional field operator for a transversely polarised gluon.

The leading helicity amplitudes have themselves power corrections, which in the case of Compton scattering are known to be of order $\Lambda^{2} / Q^{2}$, corresponding to twist four. An important milestone achieved only recently is the computation of dynamical twist-four corrections (suppressed by $m^{2} / Q^{2}$ or $\left.\boldsymbol{\Delta}^{2} / Q^{2}\right)$ for DVCS [29]. At moderate values of $Q^{2}$ these can be important in size.

The helicity of the photon and/or meson leaves an imprint on angular distributions in the final state. This provides an experimental handle to select combinations of helicity amplitudes that appear at definite order in the $\Lambda / Q$ expansion. The angular structure of the cross section is particularly rich in DVCS, because in the observable process $\ell p \rightarrow \ell p \gamma$ with $\ell=e, \mu$, Compton scattering interferes with the Bethe-Heitler process (see figure 5). The latter can be computed in QED given the input of the electromagnetic nucleon form factors, providing a unique tool to probe DVCS and thus GPDs at the amplitude level [30, 31. An analogous statement holds for TCS, $\gamma p \rightarrow \ell^{+} \ell^{-} p$.

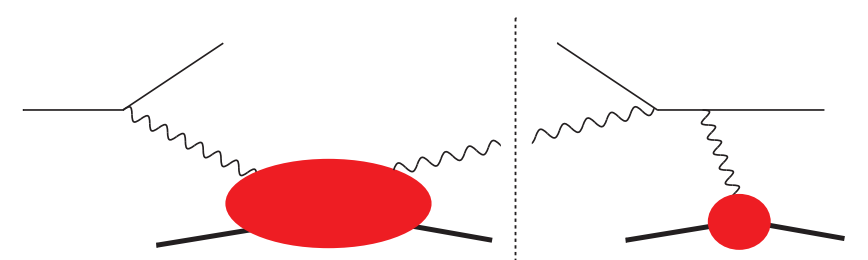

Fig. 5. Graph for the interference between Compton scattering (left) and the Bethe-Heitler process (right) in the process $\ell p \rightarrow \ell p \gamma$. The dashed line denotes the final state.

Combining the information from polarisation and angular dependence, Compton scattering can provide the most detailed access to exclusive amplitudes [32,33, and the theory to connect these amplitudes with GPDs is most precise. On the other hand, with Compton scattering alone one cannot separate the distributions for different quark flavors and has only indirect access to the gluon distributions via $\alpha_{s}$ effects. 
Meson production thus is an important source of complementary information and offers a variety of experimentally accessible production channels. Unfortunately, there is no systematic theory for power corrections in this case, whose evaluation thus requires some degree of model dependence. It remains controversial in the literature how large such corrections are in experimentally accessible kinematics, see for instance the discussion in 22, 34 and the very different analyses in [35] and [36. A detailed account of meson production is given later in this volume [37.

Let us finally mention that there are several detailed reviews on GPDs. The early development of the field and its cross connections with other areas of QCD are discussed in 38,39 and 34, a wealth of technical material is provided in 22, and [40] gives a detailed account of modelling GPDs.

\section{More on TMDs}

Let us now take a closer look at the type of factorisation that connects observables to TMDs. Focusing on unpolarised protons for the moment, the cross section for Drell-Yan production, $p p \rightarrow \ell^{+} \ell^{-}+X$ can be written as

$$
\begin{aligned}
& \frac{d \sigma}{d Q^{2} d Y d^{2} \boldsymbol{q}}=\frac{1}{s} \sum_{i j} \hat{\sigma}_{i j}(Q ; \mu) \int d^{2} \boldsymbol{k}_{1} d^{2} \boldsymbol{k}_{2} \\
& \quad \times \delta^{(2)}\left(\boldsymbol{q}-\boldsymbol{k}_{1}-\boldsymbol{k}_{2}\right) f_{i}\left(x_{1}, \boldsymbol{k}_{1} ; \zeta_{1}, \mu\right) f_{j}\left(x_{2}, \boldsymbol{k}_{2} ; \zeta_{2}, \mu\right)
\end{aligned}
$$

if the transverse momentum $\boldsymbol{q}$ of the lepton pair (and hence of the virtual photon) is much smaller than is invariant mass $Q$. The sum runs over all relevant pairs $(i j)$ of quark and antiquark distributions. Notice that in this situation, the longitudinal momentum fractions of the partons are fixed by external kinematics, with $x_{1} x_{2}=Q^{2} / s$ and $x_{1} / x_{2}=\exp (2 Y)$, where $\sqrt{s}$ is the total collision energy and $Y$ the rapidity of the lepton pair in the $p p$ centre-ofmass. This is because the condition $|\boldsymbol{q}| \ll Q$ suppresses any radiation of unobserved particles from the hard subprocess. In the more familiar case where $\boldsymbol{q}$ is integrated over, one has instead

$$
\begin{aligned}
& \frac{d \sigma}{d Q^{2} d Y}=\frac{1}{s} \sum_{i j} \int_{x_{1}}^{1} \frac{d \hat{x}_{1}}{\hat{x}_{1}} \int_{x_{2}}^{1} \frac{d \hat{x}_{2}}{\hat{x}_{2}} \\
& \quad \times \tilde{\sigma}_{i j}\left(\hat{x}_{1}, \hat{x}_{2}, Q ; \mu\right) f_{i}\left(x_{1} / \hat{x}_{1} ; \mu\right) f_{j}\left(x_{2} / \hat{x}_{2} ; \mu\right),
\end{aligned}
$$

At leading order in $\alpha_{s}$, the hard subprocess cross section is proportional to $\delta\left(1-\hat{x}_{1}\right) \delta\left(1-\hat{x}_{2}\right)$, but at higher orders hard partons are emitted into the final state. They carry away longitudinal momentum, so that one probes momentum fractions in the PDFs from $x_{1}$ or $x_{2}$ up to 1 .

The specific form (10) of TMD factorisation has been established in 41. It differs in technical details from the original version of Collins, Soper and Sterman in [42,43 (a brief account of which is given in [44). Let us highlight the role of the rapidity regulator parameters $\zeta_{1}$ and $\zeta_{2}$ in the TMDs, which satisfy $\zeta_{1} \zeta_{2}=Q^{4}$. The evolution in these parameters is simplest if we Fourier transform the TMDs from $\boldsymbol{k}$ to $\boldsymbol{z}$ (see figure 2) and then has the form

$$
\frac{d}{d \log \sqrt{\zeta}} f(x, \boldsymbol{z} ; \zeta, \mu)=K(\boldsymbol{z} ; \mu) f(x, \boldsymbol{z} ; \zeta, \mu) .
$$

The $\mu$ dependence of $f$ is given by a simple renormalisation group equation

$$
\begin{aligned}
& \frac{d}{d \log \mu} f(x, \boldsymbol{z} ; \zeta, \mu) \\
& \quad=\left[\gamma_{f}\left(\alpha_{s}(\mu)\right)-\gamma_{K}\left(\alpha_{s}(\mu)\right) \log \frac{\sqrt{\zeta}}{\mu}\right] f(x, \boldsymbol{z} ; \zeta, \mu) .
\end{aligned}
$$

More detail is given in the contribution 45 to this volume. Let us emphasise that TMDs evolve with $\zeta$ and $\mu$ at fixed momentum fraction $x$ - unlike DGLAP evolution with its interplay between the $x$ and $\mu$ dependence. This is directly related with the absence of longitudinal momentum integrals in the TMD factorisation formula (10), which we already explained. A convolution integral over longitudinal momentum occurs only when one computes the high $\boldsymbol{k}$ behaviour of TMDs, as we will see now.

At low $\boldsymbol{k}$ (or equivalently at large transverse distances $\boldsymbol{z}$ between the quark fields) the TMDs can be thought of as describing the "intrinsic" transverse momentum of partons in the proton, which arises from non-perturbative dynamics. Starting from low transverse momentum and emitting partons with large transverse momenta, one can obtain values of $\boldsymbol{k}$ much larger than the scale $\Lambda$ of nonperturbative interactions. In this situation one can express TMDs in terms of collinear PDFs and a hard-scattering kernel describing the emission. In the transverse-momentum representation, this reads

$$
\begin{aligned}
& f_{i}(x, \boldsymbol{k} ; \zeta, \mu)=\frac{1}{\boldsymbol{k}^{2}} \\
& \quad \times \sum_{j} \int_{x}^{1} \frac{d \hat{x}}{\hat{x}} C_{i j}\left(\hat{x}, \log \left(\zeta / \boldsymbol{k}^{2}\right), \log \left(\mu^{2} / \boldsymbol{k}^{2}\right)\right) f_{j}(x / \hat{x} ; \mu) .
\end{aligned}
$$

There is of course a smooth transition between the nonperturbative regime at low $\boldsymbol{k}$ and the perturbative behaviour in (14), so that there is no strict distinction between the "intrinsic" transverse momentum of a parton and the transverse momentum generated by hard radiation.

The form (14) can be used to compute cross sections in the regime of intermediate transverse momenta $\Lambda^{2} \ll$ $\boldsymbol{q}^{2} \ll Q^{2}$. Alternatively, one may describe the same physics using collinear factorisation, with the hard subprocess starting at order $\alpha_{s}$ to allow radiation of at least one parton with high transverse momentum. In fact, both descriptions give the same result at given order in $\alpha_{s}$, as suggested by figure 6] and shown for instance in 44,46. The formulation using collinear factorisation has large logarithms of $Q^{2} / \boldsymbol{q}^{2}$ in the hard-scattering cross section. Their power increases at each order in $\alpha_{s}$, which leads to a poor convergence of the perturbative series. The formulation starting from TMDs allows one to sum these so-called Sudakov logarithms to all orders in $\alpha_{s}$, using the evolution equations 


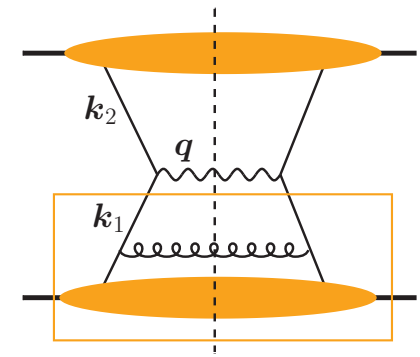

Fig. 6. A lowest-order graph for Drell-Yan production at intermediate transverse momentum of the lepton pair. The blobs represent collinear PDFs, and the box around the lower blob and gluon denotes the TMD (14) in the region of large $\boldsymbol{k}_{1}$. The decay of the virtual photon into a lepton pair is not shown for simplicity.

in $\zeta$ and $\mu$ discussed above. With reference to the original work 43, this is often called CSS resummation.

The result (14) clearly shows that the integral $\int d^{2} \boldsymbol{k}$ of a TMD requires a suitable regularisation in the ultraviolet region. For heuristic purposes, one may think of a simple cutoff in $\boldsymbol{k}^{2}$. Setting this cutoff to $\mu^{2}$ and taking the derivative with respect to $\mu^{2}$, one readily sees that the kernel $C_{i j}$ in (14) is closely related to the spitting kernel in the DGLAP evolution equations for collinear PDFs. For systematic calculations, however, one typically defines the collinear PDFs using dimensional regularisation, subtracting the ultraviolet divergence in $4-2 \epsilon$ dimensions and then setting $\epsilon=0$. The simple integral relation between TMDs and PDFs defined in 4 dimensions is then of course lost. In a modified form, it is however recovered in the Fourier conjugate representation

$$
f_{i}(x, \boldsymbol{z} ; \zeta, \mu)=\int d^{2} \boldsymbol{k} e^{i \boldsymbol{k} \boldsymbol{z}} f_{i}(x, \boldsymbol{k} ; \zeta, \mu),
$$

where the analogue of (14) reads

$$
\begin{aligned}
& f_{i}(x, \boldsymbol{z} ; \zeta, \mu)=f_{i}(x ; \mu) \\
& \quad+\sum_{j} \int_{x}^{1} \frac{d \hat{x}}{\hat{x}} \widetilde{C}_{i j}\left(\hat{x}, \log \left(\zeta \boldsymbol{z}^{2}\right), \log \left(\mu^{2} \boldsymbol{z}^{2}\right)\right) f_{j}(x / \hat{x} ; \mu)
\end{aligned}
$$

at small $\boldsymbol{z}$. The exponential in the Fourier transform indeed acts as an ultraviolet regulator for the integral, since at high $\boldsymbol{k}$ its oscillations are sufficient to give a finite result. The integral of the TMD regulated in this way gives the corresponding PDF plus corrections that can be computed in an $\alpha_{s}$ expansion. The divergence of the unregulated integral is reflected in the logarithms of $z^{2}$ on the r.h.s. of (16). It is amusing to note that for suitable functions $f(x, \boldsymbol{k})$, the exponential regulator in (15) is equivalent to a momentum cutoff 44 .

Let us now take a step back to the derivation of the TMD factorisation formula (10). This formula, and graphs like the one in figure 6 , suggest that the two protons only interact via the annihilation of a quark-antiquark pair into a virtual photon. This is barely plausible and indeed not the case. In the language of perturbation theory, the two protons can exchange an arbitrary number of soft gluons, and in addition, any number of gluons with longitudinal polarisation from each can take part in the $q \bar{q}$ annihilation subprocess, as shown in figure 7. To establish factorisation, one needs to show that these gluon interactions can be cast into a form consistent with the simple structure in (10). The result of such arguments, presented in detail in [41] (and sketched briefly in [4]), is that the physical effects of these gluons are represented by Wilson line operators between the fields in the parton correlation function (1) (integrated over $k^{-}$) and by so called soft factors, which are vacuum expectation values of further Wilson lines and can be absorbed in the definition of the TMDs. The Wilson lines also turn the operator product in (1) into a gauge invariant operator, as is appropriate for the definition of a meaningful quantity.

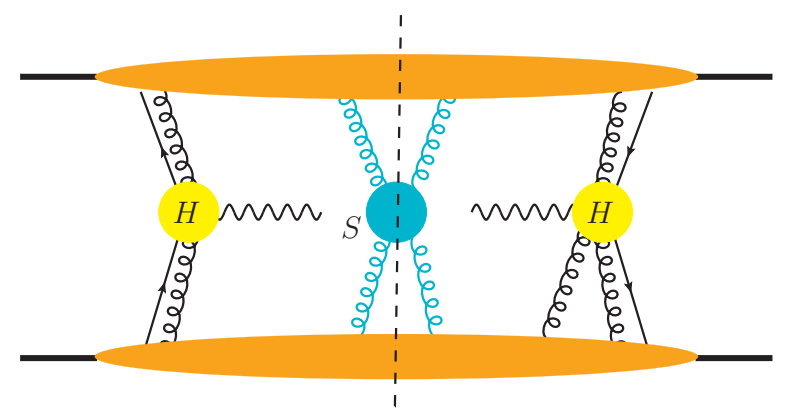

Fig. 7. Organisation of a graph for the Drell-Yan process into subgraphs that contain either hard momenta $(H)$ or soft momenta $(S)$ of momenta collinear to one of the protons (top and bottom blobs).

All this may seem to be technicalities, but indeed there is important physics behind it. The precise form of the Wilson lines allows one to regulate the rapidity divergences of TMDs, introducing a parameter $\zeta$. The associated rapidity evolution equation allows one to resum large logarithms in physical cross sections, without which one would badly fail to describe experimentally measured distributions.

A far reaching result is that the path of the Wilson lines depends on the space-time structure of the process in which the TMDs are embedded. The Wilson lines required for Drell-Yan production point to the past, whereas those appearing in the parton distributions for SIDIS point to the future. This reflects the fact that the gluon interactions shown in figure 8 strike a parton before the hard scattering in the Drell-Yan case and after the hard scattering in SIDIS.

This difference has remarkable consequences when spin dependence is taken into account. Consider the distribution of unpolarised quarks in a proton that is polarised in the transverse direction $\boldsymbol{s}$. For a proton moving in the positive or negative $z$ direction, this can be parametrised 

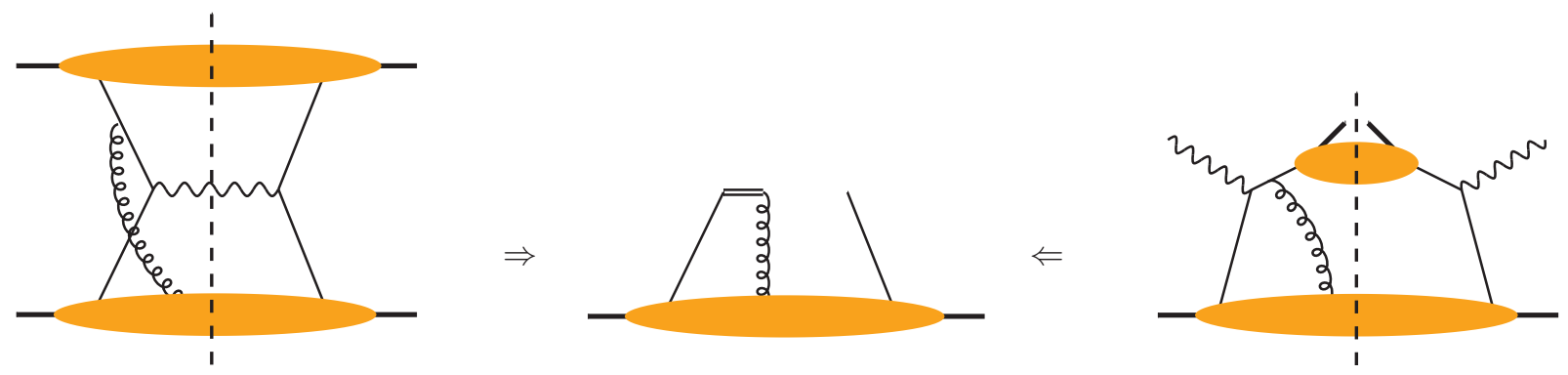

Fig. 8. Gluon exchange graphs in Drell-Yan production (left) and semi-inclusive DIS (right), as well as the corresponding Wilson line in the definition of the TMD (centre). The small blob in the SIDIS graph denotes a transverse-momentum dependent fragmentation function.

as

$$
\begin{aligned}
& f^{[U]}(x, \boldsymbol{k}, \boldsymbol{s}) \\
& \quad=f_{1}^{[U]}\left(x, \boldsymbol{k}^{2}\right)-\frac{\epsilon^{i j} \boldsymbol{k}^{i} \boldsymbol{s}^{j}}{m} \operatorname{sign}\left(P^{3}\right) f_{1 T}^{\perp[U]}\left(x, \boldsymbol{k}^{2}\right),
\end{aligned}
$$

where $\epsilon^{i j}$ is the antisymmetric tensor in two dimensions. The superscript $[U]$ indicates the Wilson line dependence; for simplicity we have omitted the arguments $\zeta$ and $\mu$ in all functions. Under time reversal the Wilson lines for DrellYan production turn into the ones for SIDIS, so that the unpolarised distribution $f_{1}$ is the same for both processes. By contrast, the factor multiplying the Sivers function $f_{1 T}^{\perp}$ in (17) changes sign under time reversal (which flips both momentum and spin vectors). Time reversal symmetry thus gives

$$
f_{1 T}^{\perp[\mathrm{DY}]}\left(x, \boldsymbol{k}^{2}\right)=-f_{1 T}^{\perp[\mathrm{SIDIS}]}\left(x, \boldsymbol{k}^{2}\right) .
$$

The modulation of the transverse-momentum distribution induced by transverse proton polarisation has opposite sign in the two cases - were it not for the gluon exchange effects represented by the Wilson line, this modulation would be zero. The Sivers distribution (as well as other spin dependent distributions that are naively zero due to time reversal invariance) shows in a pointed way that in some situations the "structure" of the proton cannot be discussed independently of the physical process in which this structure manifests itself.

For intermediate transverse momenta, $\Lambda^{2} \ll \boldsymbol{q}^{2} \ll Q^{2}$, one can also compute the graphs in the left and right panels of figure 8 using collinear factorisation. The hadronic input for the proton at the bottom of the graphs is then a twist-three distribution $T_{F}\left(x_{1}, x_{2}\right)$, called Qiu-Sterman function. The large $\boldsymbol{k}$ limit of the Sivers distribution can be expressed in terms of this function, as well. In Fourier space, the result can be cast into the form ${ }^{2}$

$$
\begin{aligned}
\int d^{2} \boldsymbol{k} e^{i \boldsymbol{k} \boldsymbol{z}} \frac{\boldsymbol{k}^{2}}{m} f_{1 T}^{\perp[\text { SIDIS }]}(x, \boldsymbol{k} ; \zeta, \mu) & \\
& =-T_{F}(x, x ; \mu)+\mathcal{O}\left(\alpha_{s}\right),
\end{aligned}
$$

\footnotetext{
${ }^{2}$ The relation (19) can be obtained from eq. (47) in 48.
}

which relates a regulated $\boldsymbol{k}$ integral of the Sivers distribution $f_{1 T}^{\perp}\left(x, \boldsymbol{k}^{2}\right)$ with the Qiu-Sterman function, in analogy to the relation (16) for unpolarised distributions. Using (18) and (19) to calculate the Sivers asymmetries in Drell-Yan production and in SIDIS, one obtains agreement with the collinear twist-three calculation at intermediate transverse momenta 46, 49. A general analysis of the relation between the two formalisms for a large class of spin asymmetries is given in 44 .

It is not always possible to describe the effects of soft gluon exchange by Wilson line operators and to obtain a factorisation formula with TMDs. Processes for which TMD factorisation has been established are SIDIS and $e^{+} e^{-}$annihilation into back-to-back hadrons (both involving transverse-momentum dependent fragmentation functions), as well as hadron-hadron collisions in which only colourless particles are produced by the hard scattering (e.g. a virtual photon, a $\gamma \gamma$ pair, a $Z$ or $W$, a Higgs boson, etc.). For hadron-hadron collisions with observed hadrons in the final state, soft gluon exchange between the two hadrons generically breaks TMD factorisation 50,51. Because soft gluon interactions cannot be reliably computed in perturbation theory, it is difficult to predict how large such factorisation breaking effects are.

\section{Spin and orbital angular momentum}

Both TMDs and GPDs have a rich structure in the parton and proton spin. They can in particular express correlations between transverse momentum or position and transverse polarisation. An example is the Sivers function we already encountered in the previous section. It is instructive to compare the transverse-momentum distribution (17) with the impact parameter distribution of unpolarised quarks in a transversely polarised proton, given by 52

$$
\begin{aligned}
& f(x, \boldsymbol{b}, \boldsymbol{s}) \\
& \quad=H\left(x, \boldsymbol{b}^{2}\right)+\frac{\epsilon^{i j} \boldsymbol{b}^{i} \boldsymbol{s}^{j}}{m} \operatorname{sign}\left(P^{3}\right) \frac{\partial}{\partial \boldsymbol{b}^{2}} E\left(x, \boldsymbol{b}^{2}\right),
\end{aligned}
$$

where $H\left(x, \boldsymbol{b}^{2}\right)$ and $E\left(x, \boldsymbol{b}^{2}\right)$ are obtained by a two-dimensional Fourier transform from the GPDs $H\left(x, \xi, \Delta^{2}\right)$ and 
$E\left(x, \xi, \Delta^{2}\right)$ at $\xi=0$. In contrast to the case of the Sivers function, the factor multiplying $E$ in (20) is not time reversal odd, because time reversal flips spins and momenta but not spatial directions. The integral of $E\left(x, b^{2}\right)$ over $x$ is related to the electromagnetic Pauli form factor of the nucleon and thus rather well known experimentally.

The decomposition (20) shows that transverse proton polarisation induces a sideways shift in the impact parameter distribution of the struck quark, which implies a shift of the spectator partons in the opposite direction (so that the overall centre of momentum remains unchanged). A fruitful idea is to relate this spatial anisotropy to an anisotropy in the transverse-momentum distribution of the struck quark: as we have already discussed, the Sivers asymmetry owes its very existence to the interactions involving the spectator partons in the proton (see figure 8). This mechanism has been called chromodynamic lensing 53 and connects in particular the sign of the Sivers distribution with the sign of the anomalous magnetic moment, in agreement with phenomenology. To quantify this relation is difficult, given the non-perturbative nature of spectator parton interactions. In specific models, using for instance perturbative gluon exchange and representing the spectator system by a diquark, one obtains however definite relations between TMDs and GPDs, as discussed for instance in [54.

Another feature of the distribution $E\left(x, \xi, \Delta^{2}\right)$ becomes evident if one changes basis from transversely polarised proton states to longitudinally polarised ones. One then finds that (in contrast to its unpolarised counterpart $H$ ), the distribution $E$ contributes to transitions that reverse the helicity of the proton while preserving the helicity of the quarks (see e.g. 34]). Since the total angular momentum $J_{z}$ along $z$ is conserved, orbital angular momentum must be transferred, which is possible because $\boldsymbol{\Delta}$ is nonzero. Specifically, one can show that $E$ involves the interference between light-cone wave functions $\psi$ and $\psi^{*}$ that differ by one unit of orbital angular momentum $L_{z}$ along $z$ [55].

A seemingly different connection between $E$ and angular momentum is given by Ji's sum rule [38,56]

$$
\begin{aligned}
J^{q}(\mu)=\frac{1}{2} \int_{-1}^{1} d x x & {\left[H^{q}\left(x, \xi, \Delta^{2} ; \mu\right)\right.} \\
& \left.+E^{q}\left(x, \xi, \Delta^{2} ; \mu\right)\right]_{\Delta^{2}=0} .
\end{aligned}
$$

Here $J^{q}(\mu)$ is the total angular momentum along $z$ carried by quarks and antiquarks of flavour $q$ in a proton polarised along $z$. It is defined via the matrix element of an appropriate angular momentum operator between proton states and includes both helicity and orbital contributions. We note that the prefactor of $E$ in (20) can be rewritten in terms of the cross product between $\boldsymbol{b}$ and the proton momentum, which is indicative of orbital angular momentum. The relation between this and the sum rule (21) is rather subtle and has been discussed in [57.

To determine from experimental information on GPDs is extremely demanding, not only because the sum rule requires extrapolation to $\Delta^{2}=0$ but also due to the prob- lem of reconstructing the $x$ dependence of GPDs (see section 3). By contrast, it is comparatively straightforward to evaluate $J^{q}(\mu)$ in lattice QCD, and there is indeed considerable activity in this direction [26, 1 .

It is important to note that there are several distinct ways to decompose the total angular momentum of the proton into contributions $J^{q}$ and $J^{g}$ from quarks and gluons, and to further separate these into contributions from orbital angular momentum and from intrinsic spin. Apart from the decomposition in 56, which is used in the sum rule (21), there is in particular the operator definition by Jaffe and Manohar [58]. Several other operator definitions have been proposed, and the contribution [1] to this volume gives a detailed discussion of this issue.

Various decompositions of angular momentum differ by terms involving the gluon field. This points to the subtle nature of gauge interactions - a theme we encountered already when discussing the role played by Wilson lines in the definition of TMDs. In a broader context, one should also remember that there may not always be a unique way to promote quantities from classical mechanics to quantum mechanics, let alone to quantum field theory. Indeed, even the definition of the quark helicity contribution the proton spin is subject to ambiguities due to the axial anomaly (see for instance 59]). The existence of such quantum effects may complicate a physical interpretation, but one should keep in mind that when calculating physical observables, a correct answer can be obtained using different schemes.

It is a pleasure to thank Andreas Schäfer and Frank Tackmann for valuable input.

\section{References}

1. K. F. Liu and C. Lorcé, arXiv:1508.00911 [hep-ph].

2. R. L. Jaffe, Lect. Notes Phys. 496 (1997) 178 hep-ph/9602236.

3. M. Burkardt and B. Pasquini, arXiv:1510.02567 [hep-ph].

4. D. E. Soper, Phys. Rev. D 5, 1956 (1972).

5. M. Burkardt, Phys. Rev. D 62 (2000) 071503, Erratum ibid. 66 (2002) 119903 hep-ph/0005108.

6. G. Watt, A. D. Martin and M. G. Ryskin, Eur. Phys. J. C 31 (2003) 73 hep-ph/0306169.

7. J. Collins and H. Jung, hep-ph/0508280.

8. J. C. Collins, T. C. Rogers and A. M. Stasto, Phys. Rev. D 77 (2008) 085009 arXiv:0708.2833 [hep-ph]].

9. I. W. Stewart, F. J. Tackmann and W. J. Waalewijn, Phys. Rev. D 81 (2010) 094035 arXiv:0910.0467 [hep-ph]].

10. I. W. Stewart, F. J. Tackmann and W. J. Waalewijn, JHEP 1009 (2010) 005 arXiv:1002.2213 [hep-ph]].

11. A. Jain, M. Procura and W. J. Waalewijn, JHEP 1204 (2012) 132 arXiv:1110.0839 [hep-ph]].

12. J. R. Gaunt, JHEP 1407 (2014) 110 arXiv:1405.2080 [hep-ph]].

13. M. Zeng, JHEP 1510 (2015) 189 arXiv:1507.01652 [hep$\mathrm{ph}]]$.

14. B. U. Musch, Ph. Hägler, J. W. Negele and A. Schäfer, Phys. Rev. D 83 (2011) 094507 arXiv:1011.1213 [hep-lat]]. 
15. S. Meissner, A. Metz and M. Schlegel, JHEP 0908 (2009) 056 arXiv:0906.5323 [hep-ph]].

16. M. Diehl and A. Schäfer, Phys. Lett. B 698 (2011) 389 arXiv:1102.3081 [hep-ph]].

17. Y. Hagiwara and Y. Hatta, Nucl. Phys. A 940 (2015) 158 arXiv:1412.4591 [hep-ph]].

18. A. V. Belitsky, X. D. Ji and F. Yuan, Phys. Rev. D 69 (2004) 074014 hep-ph/0307383.

19. R. G. Sachs, Phys. Rev. 126 (1962) 2256.

20. S. J. Brodsky, D. Chakrabarti, A. Harindranath, A. Mukherjee and J. P. Vary, Phys. Rev. D 75 (2007) 014003 hep-ph/0611159.

21. D. Müller, D. Robaschik, B. Geyer, F.-M. Dittes and J. Hořejši, Fortsch. Phys. 42 (1994) 101 hep-ph/9812448.

22. A. V. Belitsky and A. V. Radyushkin, Phys. Rept. 418 (2005) 1 hep-ph/0504030.

23. J. C. Collins, L. Frankfurt and M. Strikman, Phys. Rev. D 56 (1997) 2982 hep-ph/9611433.

24. J. C. Collins and A. Freund, Phys. Rev. D 59 (1999) 074009 hep-ph/9801262.

25. M. Vanderhaeghen, P. A. M. Guichon and M. Guidal, Phys. Rev. D 60 (1999) 094017 hep-ph/9905372.

26. Ph. Hägler, Phys. Rept. 490 (2010) 49 arXiv:0912.5483 [hep-lat]].

27. G. A. Miller, Phys. Rev. Lett. 99 (2007) 112001 arXiv:0705.2409 [nucl-th]].

28. D. Müller, B. Pire, L. Szymanowski and J. Wagner, Phys. Rev. D 86 (2012) 031502 arXiv:1203.4392 [hep-ph]].

29. V. M. Braun, A. N. Manashov, D. Müller and B. M. Pirnay, Phys. Rev. D 89 (2014) 074022 [arXiv:1401.7621 [hep-ph]].

30. X. D. Ji, Phys. Rev. D 55 (1997) 7114 hep-ph/9609381.

31. M. Diehl, T. Gousset, B. Pire and J. P. Ralston, Phys. Lett. B 411 (1997) 193 hep-ph/9706344.

32. A. V. Belitsky, D. Müller and A. Kirchner, Nucl. Phys. B 629 (2002) 323 hep-ph/0112108.

33. M. Diehl and S. Sapeta, Eur. Phys. J. C 41 (2005) 515 hep-ph/0503023.

34. M. Diehl, Phys. Rept. 388 (2003) 41 hep-ph/0307382.

35. S. V. Goloskokov and P. Kroll, Eur. Phys. J. C 53 (2008) 367 arXiv:0708.3569 [hep-ph]].

36. T. Lautenschlager, D. Müller and A. Schäfer, arXiv:1312.5493 [hep-ph].

37. L. Favart, M. Guidal, T. Horn and P. Kroll, arXiv:1511.04535 [hep-ph].

38. X. D. Ji, J. Phys. G 24 (1998) 1181 hep-ph/9807358.

39. K. Goeke, M. V. Polyakov and M. Vanderhaeghen, Prog. Part. Nucl. Phys. 47 (2001) 401 hep-ph/0106012.

40. S. Boffi and B. Pasquini, Riv. Nuovo Cim. 30 (2007) 387 arXiv:0711.2625 [hep-ph]].

41. J. C. Collins, Foundations of perturbative QCD (Cambridge University Press, Cambridge 2011)

42. J. C. Collins and D. E. Soper, Nucl. Phys. B 193 (1981) 381, Erratum ibid. 213 (1983) 545.

43. J. C. Collins, D. E. Soper and G. F. Sterman, Nucl. Phys. B 250 (1985) 199.

44. A. Bacchetta, D. Boer, M. Diehl and P. J. Mulders, JHEP 0808 (2008) 023 arXiv:0803.0227 [hep-ph]].

45. T. C. Rogers, arXiv:1509.04766 [hep-ph].

46. X. D. Ji, J. W. Qiu, W. Vogelsang and F. Yuan, Phys. Rev. Lett. 97 (2006) 082002 hep-ph/0602239.

47. M. Diehl, J. R. Gaunt, D. Ostermeier, P. Plößl and A. Schäfer, arXiv:1510.08696 [hep-ph].
48. S. M. Aybat, J. C. Collins, J. W. Qiu and T. C. Rogers, Phys. Rev. D 85 (2012) 034043 arXiv:1110.6428 [hep-ph]].

49. X. D. Ji, J. W. Qiu, W. Vogelsang and F. Yuan, Phys. Lett. B 638 (2006) 178 hep-ph/0604128.

50. T. C. Rogers and P. J. Mulders, Phys. Rev. D 81 (2010) 094006 arXiv:1001.2977 [hep-ph]].

51. T. C. Rogers, Phys. Rev. D 88 (2013) 1, 014002 arXiv:1304.4251 [hep-ph]].

52. M. Burkardt, Int. J. Mod. Phys. A 18 (2003) 173 hep-ph/0207047.

53. M. Burkardt, Nucl. Phys. A 735 (2004) 185 hep-ph/0302144.

54. S. Meissner, A. Metz and K. Goeke, Phys. Rev. D 76 (2007) 034002 hep-ph/0703176.

55. M. Burkardt and G. Schnell, Phys. Rev. D 74 (2006) 013002 hep-ph/0510249.

56. X. D. Ji, Phys. Rev. Lett. 78 (1997) 610 hep-ph/9603249.

57. M. Burkardt, Phys. Rev. D 72 (2005) 094020 hep-ph/0505189.

58. R. L. Jaffe and A. Manohar, Nucl. Phys. B 337 (1990) 509.

59. B. Lampe and E. Reya, Phys. Rept. 332 (2000) 1 hep-ph/9810270. 